\title{
Mechanical Abuse Simulation and Thermal Runaway Risks of Large-Format Li-ion Batteries
}

\author{
Hsin Wang ${ }^{1}$, Edgar Lara-Curzio ${ }^{1}$, Evan Rule ${ }^{2}$ and Clint Winchester ${ }^{2}$ \\ ${ }^{1}$ Oak Ridge National Laboratory, Oak Ridge, TN USA \\ ${ }^{2}$ Naval Surface Warfare Center, Carderock, MD USA
}

\begin{abstract}
Internal short circuit of large-format $\mathrm{Li}$-ion pouch cells induced by mechanical abuse was simulated using a modified mechanical pinch test. A torsion force was added manually at $\sim 40 \%$ maximum compressive loading force during the pinch test. The cell was twisted about 5 degrees to the side by horizontally pulling a wire attached to the anode tab. The combined torsioncompression force created small failure at the separator yet allowed testing of fully charged large format $\mathrm{Li}$-ion cells without triggering thermal runaway. Two types of commercial cells were tested using 4-6 cells at each state-of-charge (SOC). Commercially available $18 \mathrm{Ahr} \mathrm{LiFePO}_{4}$ (LFP) and $25 \mathrm{Ahr} \mathrm{Li}(\mathrm{NiMnCo})_{1 / 3} \mathrm{O}_{2}$ (NMC) cells were tested, and a thermal runaway risk (TRR) score system was used to evaluate the safety of the cells under the same testing conditions. The aim was to provide the cell manufacturers and end users with a tool to compare different designs and safety features.
\end{abstract}

Keywords: Large format Li-ion battery, mechanical abuse, pinch-torsion, thermal runaway risk Corresponding author e-mail address: wangh2@ornl.gov, Tel: 865-576-5074, FAX: 865-5746098 


\section{Introduction}

Internal short circuit and subsequent thermal runaway have been important safety issues for the Li-ion batteries used in cell phones and laptop computers. In small electronic appliances, the estimated occurrence of an internal short circuit and thermal runaway is one in 5 million or even smaller. Many attempts have been made to simulate the internal short circuit which is believed to be caused by manufacturing defects or lithium dendrite growth during operation [1]. In practice, all the field events of thermal runaway left little evidence on what actually happened because the high temperatures during thermal runaway consumed most cell components. It is therefore hard to pin-point the origin of the internal short circuit. Despite the concerns for thermal runaway, small Li-ion batteries are generally considered safe and can even be carried onto airplanes. This is because the total number of cells in a pack is small (4-6 cell packs for laptops), and individual cell capacity ( $<3 \mathrm{Ahr})$ is small in electronic appliances such as a smart phone. UL 1642 [2] addresses the safety issues and testing procedures of the small Li-ion cells.

The high energy density, good power density and high charge/discharge efficiency of Li-ion batteries have made electrical vehicles a practical alternative to internal combustion engine vehicles. The automotive manufacturers have made hybrid electric vehicles (HEV) since 1997, and most of them are employing Li-ion battery systems. Electrical vehicles (EV) and extended range electrical vehicle (EREV) have been available in the market since 2011. The introduction of electric vehicles into the market also made the battery safety a higher priority. Unlike Li-ion cells in the consumer electronic market, in which a thermal runaway event may only cause a small hot gas release and controllable fire, a battery failure in an electric vehicle can be catastrophic. 
The increasing safety risks of large format Li-ion cells are due to the higher capacity of individual cells (15-32.5 Ahr) and the large number of cells used in a pack. The amount of energy release caused by mechanical damage from a fully charged cell can result in a much higher local temperature rise followed by thermal runaway. Even with improved safety under non-collision conditions the cell behavior during and after a collision by mechanically induced internal short circuit is critical. With increasing number of electrical vehicles on the road, auto accidents involving EVs are inevitable. Without a reliable cell safety test of large format batteries, the current strategies are:

1. Mechanically isolate the battery pack and use heavy and strong armors to protect the cells from deformation

2. Development of alternative cell chemistry with higher onset of thermal runaway temperatures

3. Limiting the battery operation range, i.e. a smaller window of state-of-charge (SOC), to stay below full capacity

There is no widely accepted mechanical abuse cell safety test available, especially for thermal runaway of large format $\mathrm{Li}$-ion cells. Unlike the small consumer electronic cells, large format cells can easily undergo thermal runaway due to a mechanically induced short circuit. It is very difficult to establish a standard test in which pass and fail are simply defined by fire or smoke. The goal of this study is to develop a reliable mechanical abuse test for large format Li-ion cells and demonstrate the test is capable of distinguishing cell safety risks. For large format cells, we are no longer trying to simulate the small manufacturing defects. The aim is to find a sensitive enough test that different large format cells can be compared. It is not realistic for this test to be 
a PASS/FAIL test that simulates the internal short circuit induced by mechanical damages as a result of car crashes because damage area in every crash could be different. What the test can provide is a relative risk comparison of different cells. In the absence of other testing or information on safety, knowing the relative safety risk of the cells that involved in an accident is valuable information to first responders and such as fire fighters and tow truck operators. The pinch test developed at Oak Ridge National laboratory (ORNL) has been modified, and a pinchtorsion test was developed for large format cells utilizing battery safety testing facilities at the Naval Surface Warfare Center (NSWC). Preliminary results and analysis are presented.

\section{Mechanical Abuse Tests and Internal Short Circuit}

\subsection{Mechanical Abuse Tests}

Various experiments have been designed to simulate the internal short circuit. The majority of the tests were mechanical abuse tests in nature. The goal for internal short circuit simulation is to create a small contact point inside a cell, preferably between two electrodes and the separator. However, to simulate a realistic contact short circuit the damage to the cell must be kept at minimum and the local cell chemical and microstructure must be kept unchanged. These are the main challenges to successful simulations of internal short circuit. For mechanical abuse tests that used to assess the cell thermal runaway risk, minimal damage to the cell is still preferred. There are many ways to create internal contact inside a cell. The following methods can be found in the literature:

Nail Penetration Test: The most noticeable battery safety test is the nail penetration method [3] in which a nail is forced into the cell causing short circuit. The results of nail test have been 
mixed and inconclusive. The penetration of the cell casing and metal-to-metal contact with multiple layers inside the cell made it a more severe test than the intended small defect that is believed to cause internal short circuit. In this case, a cell can be mislabeled as unsafe because it failed a harsh test. On the other hand, the nail is acting as a heat sink that can remove local heating much quicker and may led to an unintended pass of the test.

Small Indentation Test: A modified version of the nail penetration test is the small indentation test, used by Underwriters Laboratory for cell safety test (UL 1642) and also adopted by the USABC group $[4,5]$. In this test, a blunt metal pin with $3 \mathrm{~mm}$ radius is used to mechanically indent the cell. Usually a load frame is used with controlled loading speed and a furnace is used to perform the test at various temperatures. It is by far the most successful test for cylindrical cells. However, in prismatic cells the failure tends to happen near the contact surface and mechanical fracture of the can and pouch often occurs. This test is currently limited to small capacity cells $(<3 \mathrm{Ahr})$ and not widely used for large format cells.

BAJ Test: The Battery Association of Japan (BAJ) suggested an alternative internal short circuit test [6] in which the jelly-roll of a charged battery is taken out and a small metal particle is placed under the unwrapped layers. The cell is then subjected to mechanical pressure until a short circuit occurs. This procedure requires disassembly of the live cells and is not practical for the industry to adopt.

Implanted Instigators: There are several other internal short circuit tests that require a foreign object to be implanted inside the cell during manufacturing. They are proposed by Sandia 
National Laboratory (SNL) [7] using Wood's metal, a eutectic alloy of 50\% bismuth, 26.7\% lead, $13.3 \%$ tin, and $10 \%$ cadmium by weight with a melting point of $70^{\circ} \mathrm{C}$, National Renewable Energy Laboratory (NREL) using a phase-change material [8]. In some tests [9-11] an external field is used to induce the internal short circuit. The filed can be electrical, ultrasonic or magnetic. The main issue of these approaches is the requirement of manufacturers to put a foreign object inside the cell. It is not a practical approach for the end users to evaluate the cells from various suppliers.

\subsection{Mechanical Pinch System}

A mechanical pinching test was developed at ORNL by integrating the load frame with precisely controlled compression using two spheres [12-13]. The speed of the test can be as low as a few microns per second. Failure usually occurs below the top layers and the contact area can be 1-2 $\mathrm{mm}$ in diameter. The major drawback is this test only works for prismatic cells. The initial ORNL pinch test system was a MTS servo-hydraulic machine. This system is capable of testing prismatic cells up to $3 \mathrm{Ahr}$. The cell voltage was monitored during the pinch test, and the detection threshold was set at $0.1 \mathrm{~V}$ at which the loading was reversed at $10 \mathrm{x}$ the pinch speed. This test was shown to be sensitive enough to distinguish cells with different designs.

The important features of the pinch test are the characteristic voltage drop and temperature responses. It is a simple pass (positive) and fail (negative) test:

- A negative response (thermal runaway) is a rapid voltage drop followed by no recovery and a large temperature increase in the cell to $>150{ }^{\circ} \mathrm{C}$. 
- A positive response (safe cell) is a sharp voltage dip followed by a voltage recovery and a small temperature increase, typically below $100^{\circ} \mathrm{C}$, but can sometimes reach below 130$150^{\circ} \mathrm{C}$ reaction triggering temperature depending on specific cell chemistries

The short circuit site produced by the pinch-only test was still too large for some cells, especially the one with higher energy density. It was further improved with the addition of torsion components during compression [14-15]. Instead of simple compressive loading, torsion was added to simulate driving a screw driver into the cell. The torsion was able to cause separator failure much earlier and create a smaller contact area. The subsequent short circuit was less severe allowing some large format cells to be charged to $100 \%$ SOC.

\subsection{Large Format Cell Test System}

Commercially available large format Li-ion cells (18 Ahr LFP cells and 25 Ahr NMC cells) were tested using the pinch method at NSWC in Carderock, MD. These two types of cells have similar size electrodes, overall weight and density. Both cells were manufactured and assembled under similar conditions. In terms of capacity, a $72 \%$ SOC NMC cell is comparable to a $100 \%$ SOC LFP cell. Although it is ideal to have cells that have the same chemistry and the same capacity, and carry out testing with a larger scale test matrix, we focused on developing a test that sensitive enough to compare these two types of cells. The test chamber was a reinforced steel structure equipped with a high-efficiency ventilation system designed to handle the smokes and fire of large battery modules and packs. A LabView based data acquisition system was used to monitor and record the cell voltage and temperature. A FLIR A325 infrared camera was placed inside the chamber to monitor the cell surface temperatures. The chamber was equipped with video recording and real-time monitoring. In order to improve parameter control a motor-driven load 
frame was built. The system used a 5,000 pound load cell. The actuator moved at $0.001 \mathrm{inch} / \mathrm{min}$ or faster. We used test speeds at $0.01 \mathrm{in} / \mathrm{min}$ and $0.05 \mathrm{in} / \mathrm{min}$. It has been shown that the loading rate does not affect the load vs. displacement within the test speed range. Cell voltage was used as the trigger signal and a voltage drop indicated the onset of internal short circuit. The system sensitivity was set typically at $\Delta \mathrm{V}=0.1 \mathrm{~V}$, and the pinch head would return at $1 \mathrm{inch} / \mathrm{min}$ upon the detection of a failure.

From previous work of small capacity prismatic cells [18], it was clear the pinch-only test induced too much damage to the cell; it was beneficial to introduce a torsion component during the compression. While such testing is available in commercial hydraulic testing systems such as MTS, adding a torsion force in the portable system requires a second motor and was not practical for the limited space inside the test chamber. The pinch-only tests put cells under compression. It took large compressive force (about 1500-1700 lbs) and large deformation (70$80 \%$ of total cell thickness) to produce a mechanical failure in the separator. The excessive mechanical damage to the cells during pinching was the main reason for higher rate of thermal runaway in fully charged Li-ion cells. The pinch-only test shown in Figure 1 was modified to introduce a torsion component manually. A manual pulling mechanism was accomplished by attaching a wire to the mechanically stronger anode tab made of a $\mathrm{Cu}-\mathrm{Ni}$ alloy (elastic modulus $152 \mathrm{GPa}$ ) compare to the aluminum cathode tab (elastic modulus $69 \mathrm{GPa}$ ). The cell was pulled horizontally to "twist" the cell about 5 degrees after at least $600 \mathrm{lbs}$ of compressive force. This modification allowed some large-format cells to be tested to $100 \%$ SOC without thermal runaway and demonstrated a practical method to evaluate large-format cells experiencing mechanical abuse. 


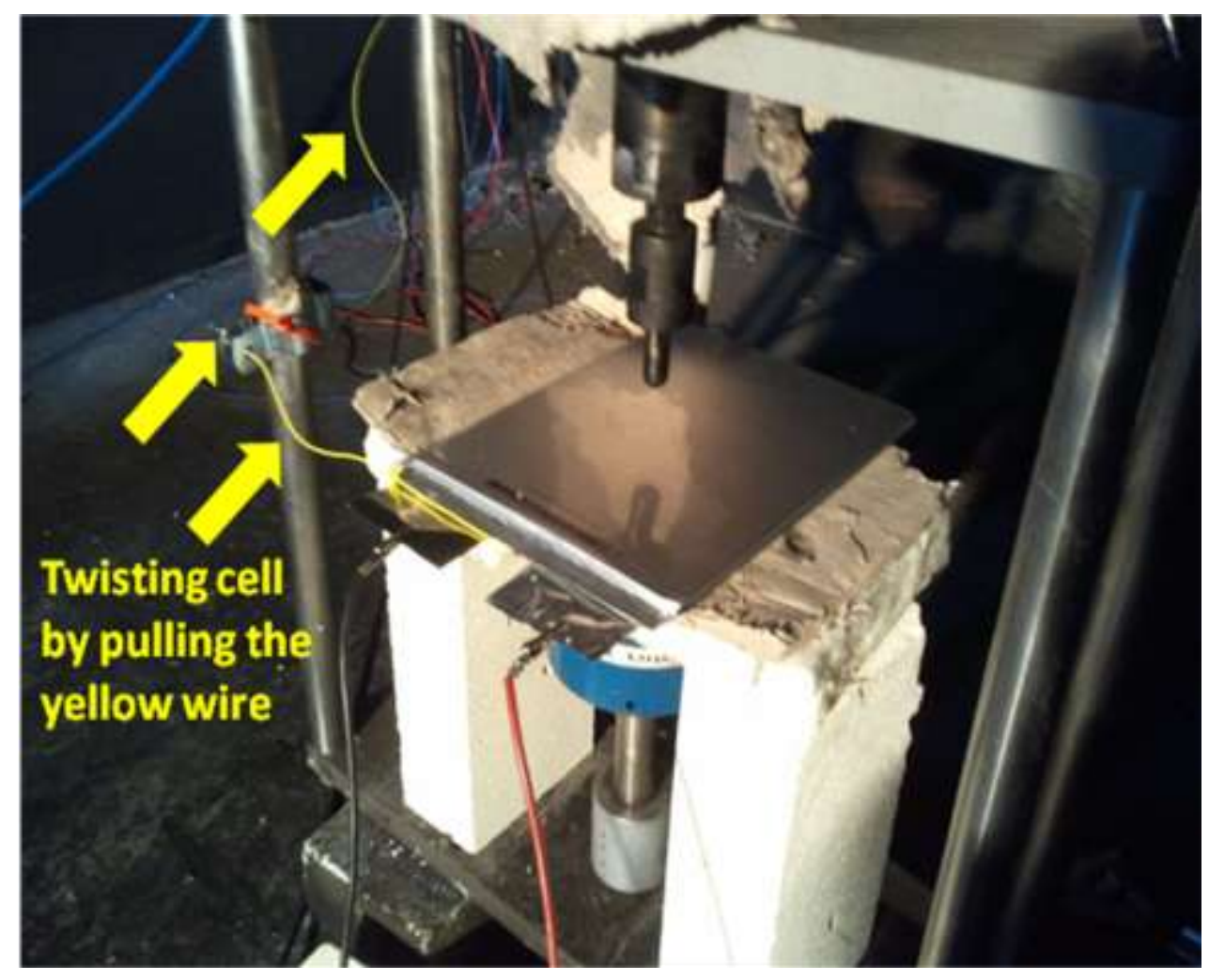

Figure 1. Modified pinch-twist test: The cell can be twisted by pulling the yellow wire attached to the anode tab

\section{Results and Discussion}

\subsection{Large-format 18 Ahr Lithium Iron Phosphate (LFP) cells}

Large format LFP cells were purchased from a commercial source with dimensions of 161 $\mathrm{mm} \times 230 \mathrm{~mm} \times 6.5 \mathrm{~mm}$, and each cell weighted $490 \mathrm{~g}$. The nominal cell voltage was $3.2 \mathrm{~V}$ and maximum capacity was $18 \mathrm{Ahr}$. The energy density without the tab was $240 \mathrm{Whr} / \mathrm{L}$. 
Since the SOC of LFP cells were difficult to determine from the open circuit and discharge voltage, the cells were all first charged to full capacity and for each condition, a fixed portion of full capacity was removed for lower capacity tests.

For the LFP cells, a typical positive cell response is shown in Figure 2, in which a quick voltage drop was accompanied by a fast partial recovery after the pressure was released. The test speed for the LFP cells was at $0.05 \mathrm{inch} / \mathrm{min}$ and all the pinch-only tests were very repeatable with failure occurring at the same displacement values and compressive load 1.2

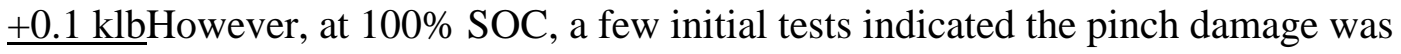
excessive. Most cells would go to thermal runaway because of higher current and local temperature rise. The torsion component was added during the pinch test as described in Figure 1. A comparison of pinch-only tests and pinch-torsion tests are shown in Figure 3. The pinch-only test, Figure 3(a), showed a very fast voltage drop followed by a discharge to zero voltage within 1-3 minutes after the compressive pressure was released. Thermal runaway occurred as a result. The pinch-torsion test on another cell with the same capacity, Figure 3(b), was able to create the characteristic voltage drop-and-recovery without thermal runaway. 


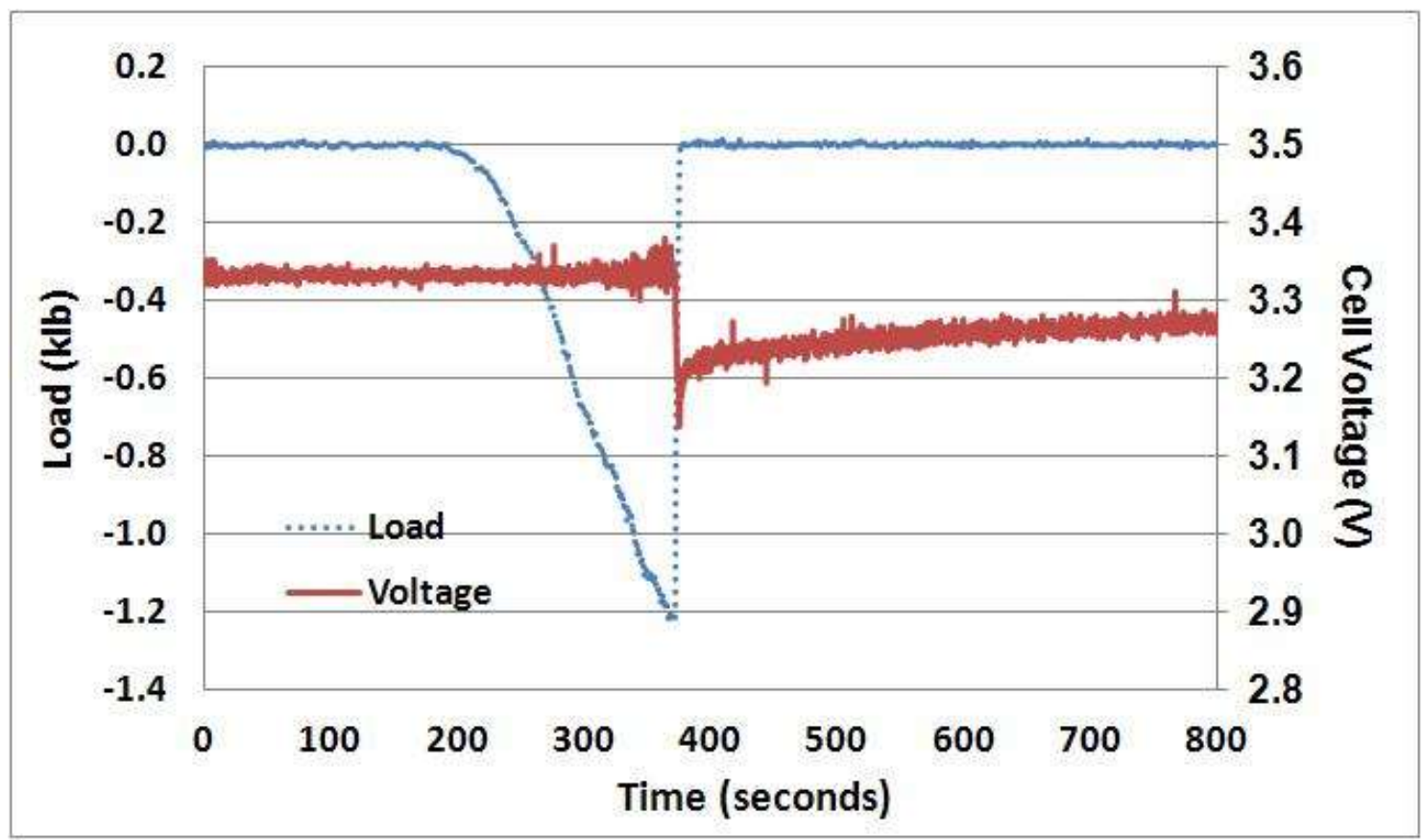

Figure 2. Characteristic voltage drop-and-recovery indicating a successful creation of internal short circuit.
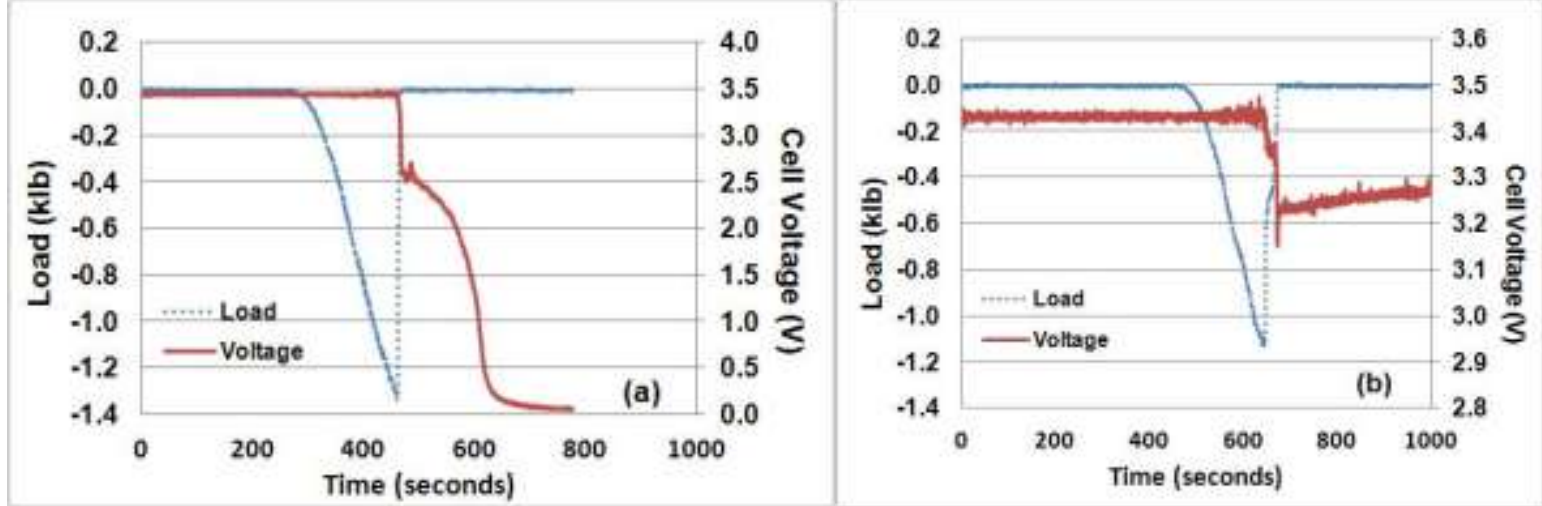

Figure 3. (a) Pinch-only test of a 100\% SOC cell resulted in rapid voltage drop followed by thermal runaway; (b) Pinch-torsion test on $100 \%$ SOC cell resulted in a voltage drop-and recovery (no thermal runaway) 


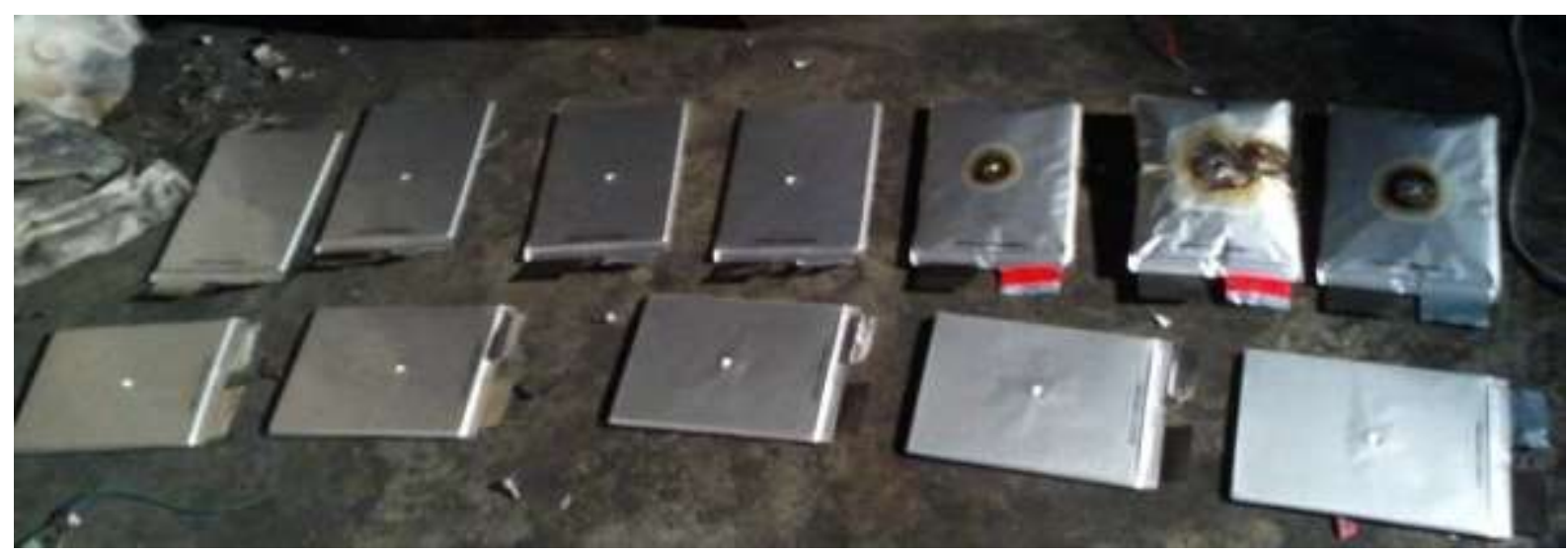

Figure 4. Twelve LFP cells tested: three with burn marks (from the top right corner) were Pinch-only and 9 other cells were Pinch \& Twist tests

In Figure 4, a total of 12 cells are shown after the tests. The three cells with burn marks were tested with pinch-only. There were excessive damages to the pouch, and the cell released a large amount of heat and some with smoke. Temperatures of the three cells reached $150{ }^{\circ} \mathrm{C}$ or higher within 3-5 seconds follow by release of smoke and hot gas. The other nine cells were tested with a torsion force added after $600 \mathrm{lbs}$ initial compressive force. Five cells were charged to $90 \%$ SOC and four cells were charged to $100 \%$ SOC. All the cells tested with pinch-torsion failed at a smaller compressive force than the pinch-only tests. More importantly, the voltage drop showed typical recovery, and no thermal runaway was observed. The test details are listed in Table 1. After testing cells at 90\% and 100\% SOC, no additional LFP cells were tested at lower SOC because no thermal runaway was likely to occur. The ability to test 18 Ahr cells at $100 \%$ SOC made it possible to access thermal runaway risks of large-format cells used for electric vehicles. Under the system developed to assess the thermal runaway risks, the 18 Ahr LFP cells had a perfect safety score of 100, which also meant there was $0 \%$ chance of thermal runaway under these test conditions. 
Table 1. LFP cells tested at $100 \%$ and $90 \%$ SOC.

\begin{tabular}{|c|c|c|c|c|c|c|c|}
\hline Cell & $\begin{array}{c}\text { Voltage } \\
(\mathrm{V})\end{array}$ & $\begin{array}{c}\text { Capacity } \\
\text { (Ahr) }\end{array}$ & SOC \% & Number & Number of & Safety & Thermal \\
of Tests & $\begin{array}{c}\text { Scrmal } \\
\text { runaway }\end{array}$ & Runaway \\
Risk
\end{tabular}

\subsection{Large-format 25 Ahr Nickel, Manganese, Cobalt (NMC) Li-ion Cells}

In order to compare different cells, large-format NMC cells were purchased from the same commercial source with dimensions of $161 \mathrm{~mm}$ x $230 \mathrm{~mm}$ x $6.0 \mathrm{~mm}$ and each cell weighted $485 \mathrm{~g}$. The nominal cell voltage was $3.65 \mathrm{~V}$, and maximum capacity was $25 \mathrm{Ahr}$. The energy density without the tab was $410 \mathrm{Whr} / \mathrm{L}$. To be consistent with LFP cells testing the NMC cells were all discharged from shipping voltage and charged to full capacity of $25 \mathrm{Ahr}$. For each condition, $8-50 \%$ of full capacities were removed from the cell. Since the cell dimensions and weight were similar to the LFP cells, the same pinch-torsion tests were carried out to evaluate the NMC cells. Since the initial tests of higher capacity cells resulted in thermal runaway the test speed was slowed down to $0.01 \mathrm{inch} / \mathrm{min}$ to better monitor the failure events. Loading speed was shown not a factor for either the LFP cells or the NMC cells. Figure 5 are typical cell responses of NMC cells at various SOC. Four NMC cells charged to $50 \%$ SOC were subjected to the pinch-twist test. All four cells showed the positive responses with voltage drop-and-recovery as Fig. 5(a). 


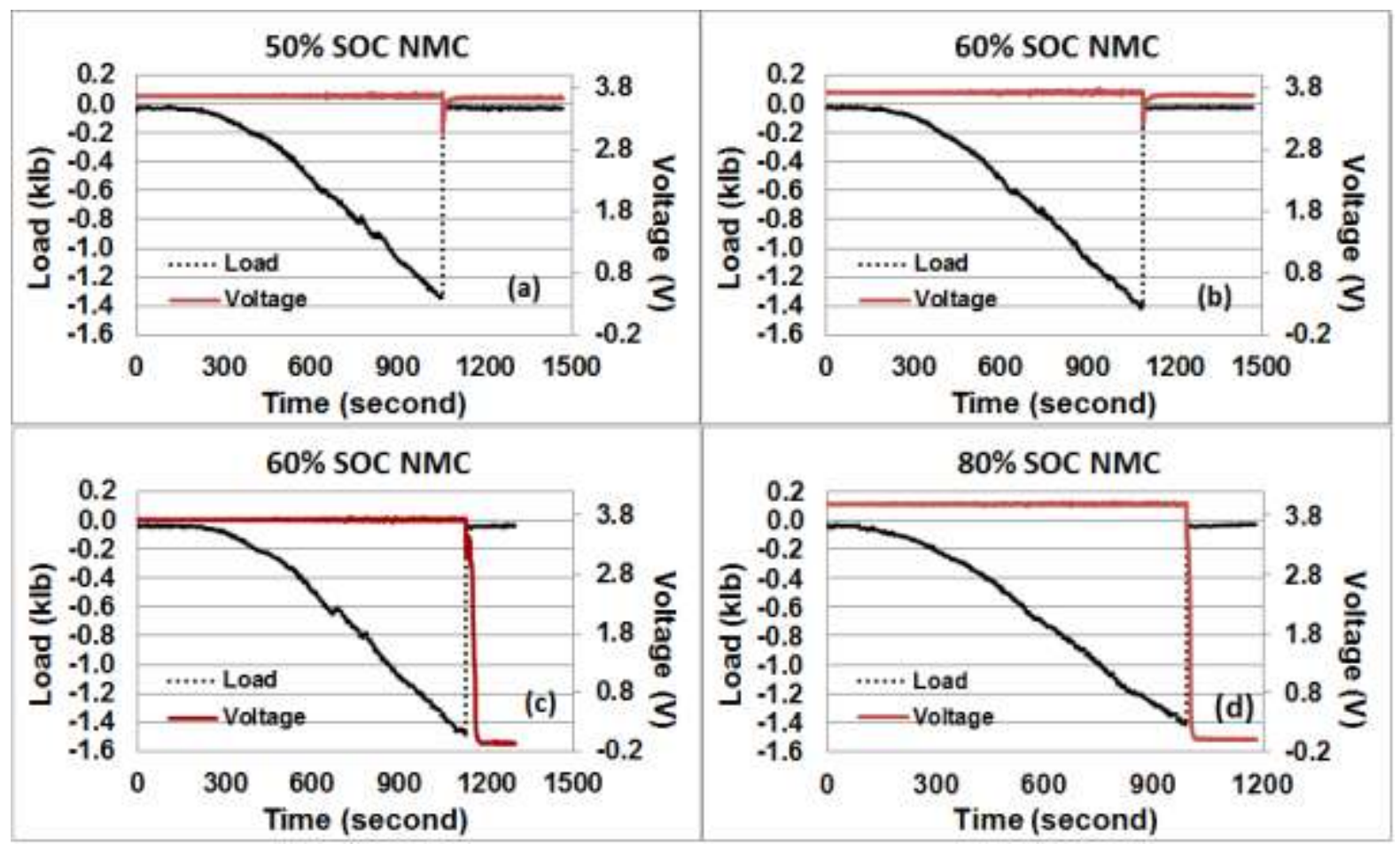

Figure 5. Load and Voltage vs. Time plots of four $25 \mathrm{Ahr}$ NMC cells at a) $50 \% \mathrm{SOC}, \mathrm{V}_{\mathrm{OC}}=$ $3.65 \mathrm{~V}$; b) $60 \% \mathrm{SOC} ; \mathrm{V}_{\mathrm{OC}}=3.72 \mathrm{~V}$ c) $60 \% \mathrm{SOC} ; \mathrm{V}_{\mathrm{OC}}=3.73 \mathrm{~V}$ and d) $80 \% \mathrm{SOC} ; \mathrm{V}_{\mathrm{OC}}=$ $3.90 \mathrm{~V}$.

A total of six cells charged to $60 \%$ SOC were tested and three out of six cells showed positive responses with voltage drop-and-recovery, shown in Figure 5(b). Three other cells showed cell voltage recovered briefly and quickly dropped to $0 \mathrm{~V}$ followed by a thermal event with pouch out gassing and smoke, as shown in Figure 5(c). At 50\% chance of thermal runaway, we determined $60 \%$ SOC was the threshold of thermal runaway for the $25 \mathrm{Ahr}$ NMC cells. In order to confirm cells with higher SOC than $60 \%$ would definitely go to thermal runaway under the same test conditions, two cells at $80 \%$ and $92 \%$ SOC were tested. The Voltage and Load vs. Time curves of the $80 \%$ SOC cell are shown in Figure 5(d). The voltage dropped to zero volt with no bounce back and the subsequent thermal runaway observed by IR camera showed a steep temperature rise slope (described in section 3.3) with more visible gas release and smoke. The $92 \%$ SOC cell had similar responses followed by 
thermal runaway. No more cells at higher SOC than $60 \%$ were tested because the results clearly showed $60 \%$ SOC is the limit.

\subsection{Temperature Changes of the 18 Ahr LFP and 25 Ahr NMC Cells}

An infrared camera was used to record the surface temperatures of cells during all the tests. It provided digital temperature maps of the entire cell surface and the thermal event could be played back of frame-by-frame. Figure 6 shows four typical Temperature vs. Time profiles from an area right next to the indenter on the LFP and NMC cells. At 100\% SOC, the LFP cell with pinch-torsion showed a gradual temperature rise and stabilized after 30 seconds at $60{ }^{\circ} \mathrm{C}$. However, the LFP cell charged to $100 \%$ SOC experienced pinch-only test went to thermal runaway within 3-5 seconds, surface temperatures quickly exceeded the IR camera setting of $150^{\circ} \mathrm{C}$ and dropped back within the range after 90 seconds. For the $25 \mathrm{Ahr}$ NMC cells, pinch-torsion tests were used for all the cells. Even at low SOC of 50\%, all the cells would go to thermal runaway for the pinch-only test. Although the voltage recoveries in Figure 5 (a) and (b) indicated the short circuit was brief and the cells recovered quickly, the temperature profiles showed a very different response between the LFP and NMC cells. As shown in Figure 6, the 50\% SOC NMC cell had a sudden, very local temperature rise of 120$130{ }^{\circ} \mathrm{C}$ and quickly cooled down to $55^{\circ} \mathrm{C}$ within 60 seconds, during which the cell average temperature remained below $60^{\circ} \mathrm{C}$. The IR images also confirmed a localized temperature spike that was contained. The energy was not enough to trigger thermal runaway throughout the cell. However, at $80 \%$ SOC, the temperature quickly exceeded $150^{\circ} \mathrm{C}$ and thermal runaway was triggered. Compare to the slow rise to $60^{\circ} \mathrm{C}$ of the LFP cell, the NMC cells had more energy release after the short circuit. The thermal responses of the two types of cells 
were associated with the total available energy and different cell chemistries.

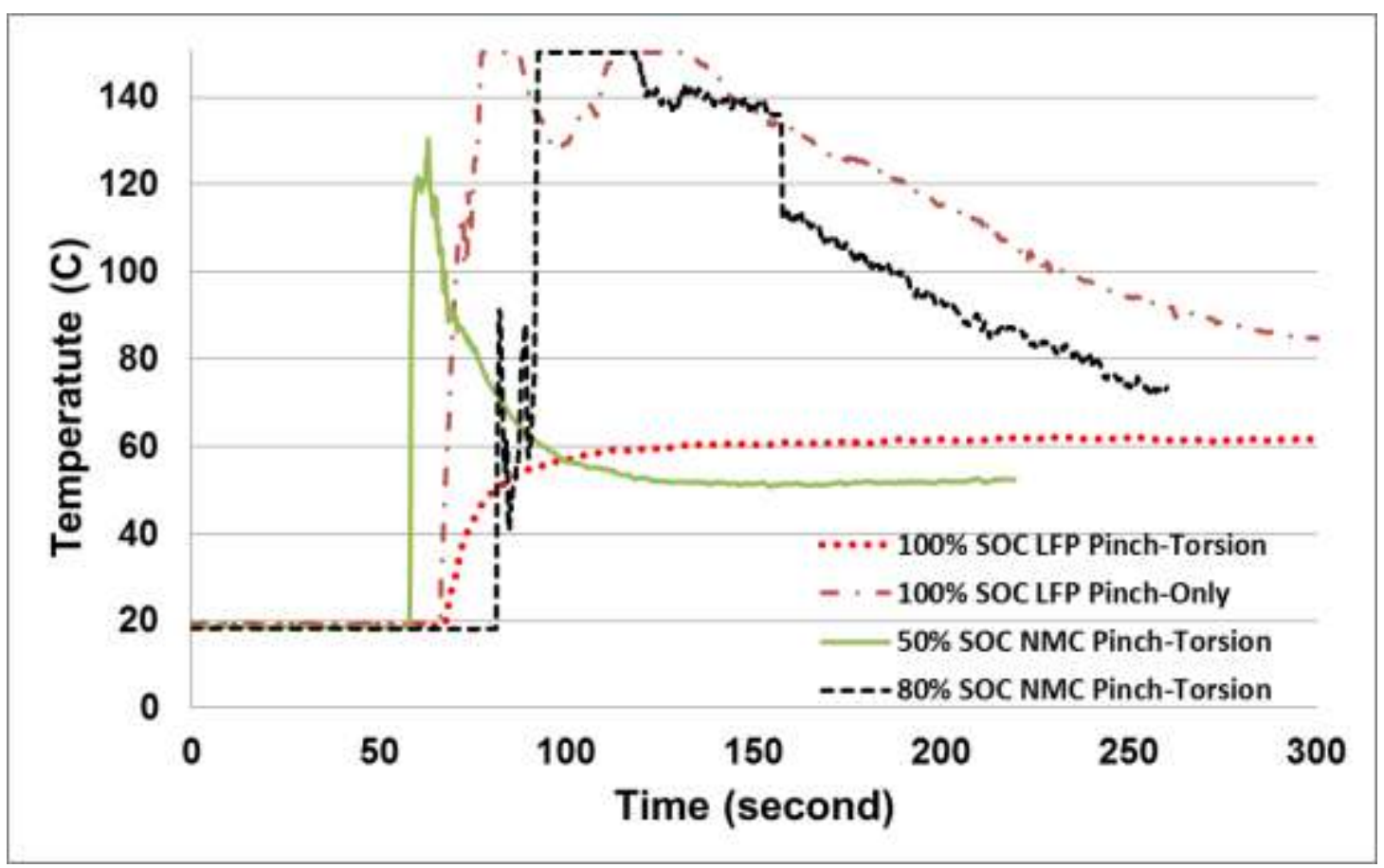

Figure 6. Temperature vs. Time plots of LFP and NMC cells showing various thermal responses under different loading modes and state of charge (SOC)

The IR camera also captured the details of cell temperature evolution right after the short circuit, as shown in Figure 7 for a 60\% SOC NMC cell that went to thermal runaway. Instead of a typical heat transfer isotherm spreading from the pinch location, the temperature went up to $147.5^{\circ} \mathrm{C}$ within 0.5 second and showed an irregular thermal pattern extending in several directions. This pattern was similar to the ignition of fire when lighting a match or a gas burner with flames shooting out.. The IR images were taken every 0.25 second. The rapid ignition-style heat spread was much faster than the thermal conduction speed. It was a clear indication of local heating could not be contained and triggered exothermic chemical reactions. 

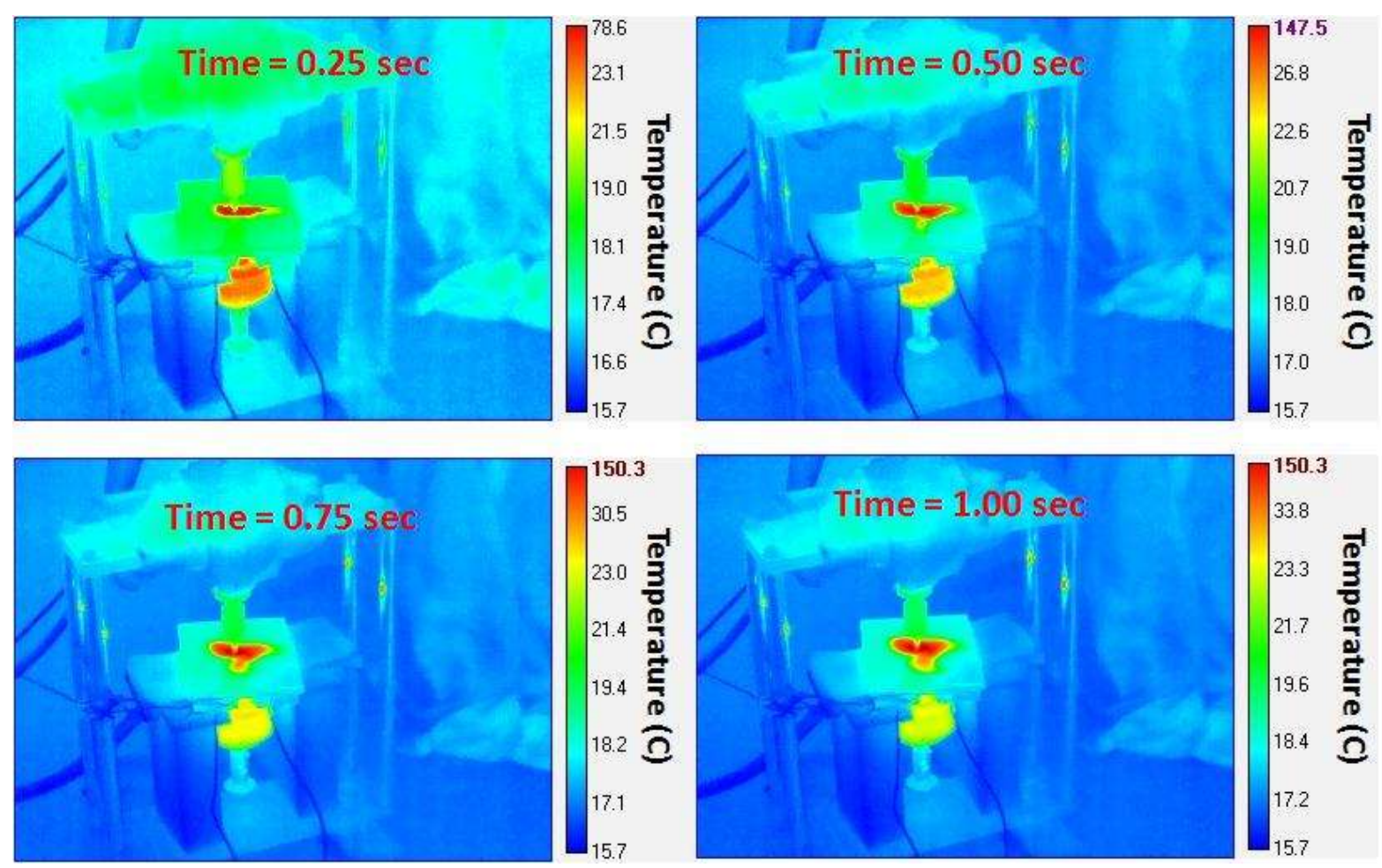

Figure 7. $25 \mathrm{Ahr}$ NMC cell at $60 \%$ SOC before thermal runaway (color bar automatically adjusted to the maximum and minimum temperatures
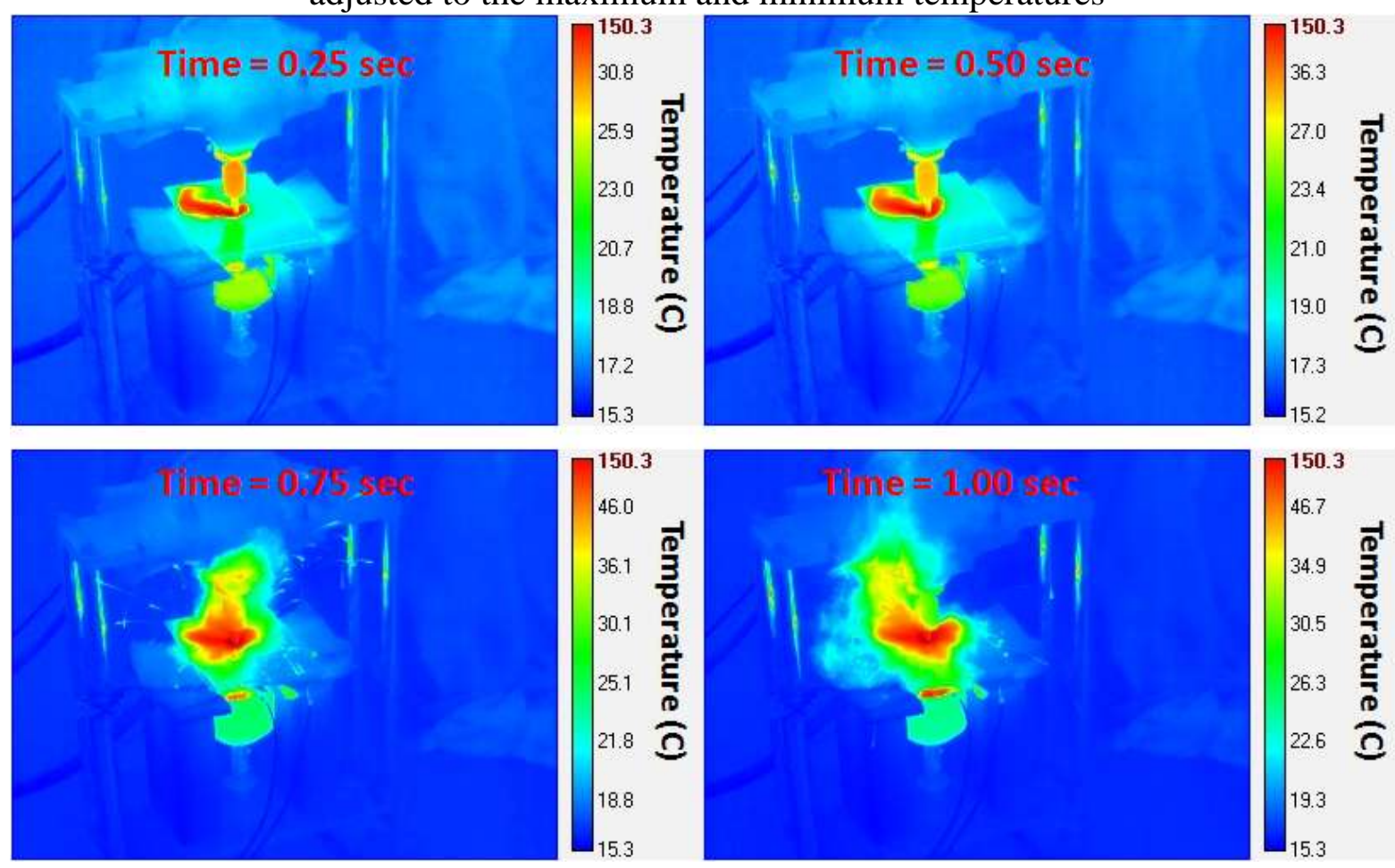

Figure 8 . Four IR images of a $25 \mathrm{Ahr}$ cell charged to $80 \%$ SOC before thermal runaway 
In Figure 8, the IR images of the 80\% SOC NMC cell right after short circuit were shown. During the first second ( $4 \mathrm{~Hz}$ frame rate), the temperature went up to $150^{\circ} \mathrm{C}$. The irregular flame-like temperature pattern spreading to the left could be seen in the two top images. The next two images showed hot gas release and thermal runaway. Compared to the $60 \%$ SOC cell in Figure 7, the extra $20 \%$ energy resulted in higher local temperatures $\left(76.8^{\circ} \mathrm{C}\right.$ vs 150 ${ }^{\circ} \mathrm{C}$ at 0.25 second) and faster triggering of the chemical chain reactions.

\subsection{Safety Evaluation of Large Format Li-ion Cells}

We have shown that for large format cells, mechanical pinch-torsion can be effectively used to induce a small local short circuit site inside the pouch cells. By monitoring and recording the Voltage and Load vs. Time curves, typical cell responses can be captured for safety evaluations. Temperature monitoring of the cells during testing provides extra information and is always helpful to the analysis. Although an IR camera provides surface temperature distribution, it may not be an economical and practice option. The minimum requirement of a surface mounted thermocouple is recommended. The $25 \mathrm{Ahr}$ NMC cells were failing at $50 \%$ ratio (3 out of 6 cells) when charged to $60 \%$ SOC. They could survive at $50 \%$ SOC with no failure; i.e. thermal runaway did not occur and the charge partially recovered. Compared to the 18 Ahr LFP cells which had $100 \%$ survival rate at $100 \%$ SOC, it was clear which type of cells were more prone to thermal runaway under same testing conditions. It is also important to point out that the NMC cells failed because the local heat generation was too much. It means an even smaller short circuit site is needed to keep the heating local. 
There is still room to improve the pinch-torsion test to allow higher capacity cells to be tested without going to thermal runaway. For example, the torsion applied to the cell was accomplished by manually pulling a wire and creating a one-time twist during pinching. An automated bi-axial loading system would provide more control to induce smaller size failure in the separator and more consistent internal short circuit events. Other important factors that could affect the test repeatability and sensitivity are data acquisition speed and voltage drop detection limit. Since the pinch or pinch-torsion test is a continuous deformation process, at various speeds the particles in the electrodes that initiated the short circuit through the failed separator could move away from the shorted spot. In a sensitive system, a smaller "soft short", i.e. $\Delta \mathrm{V}=1-10 \mathrm{mV}$, and recovery could be detected. In this demonstration work, the test sensitivity of voltage drop was set at $\Delta \mathrm{V}=100 \mathrm{mV}$ due to the noise level in the voltage channels. Signal quality and data acquisition system could be further improved. As the SOC increases more energy could be released after the short circuit. The cell response would progress into irreversible discharge (deeper voltage drops) and higher local temperatures. Therefore, the occurrence of the small voltage drop followed by instantaneous recovery under load diminished as the cells were charged to higher voltages (or higher capacities). For certain cells, even after the removal of the load, the local temperature could rise above the onset of exothermic reactions and lead to thermal runaway. The key of a sensitive mechanical abuse test is to set the voltage-drop at the right level so significant number of cells with better thermal management design or improved chemistry (with higher on-set thermal runaway temperatures) will exhibit a typical "soft short" voltage drop-andrecovery response at $100 \%$ SOC. In the meantime, some of the cells with different designs or chemistries would go to thermal runaway at $100 \%$ SOC but behave "safer" at reduced SOC. 
While this work demonstrated important parameters for the pinch-torsion method, the numbers of cells tested were limited and further optimization is needed. The exact testing protocols may be determined by organizations or groups setting test standards.

For the NMC cells, the results are summarized in Table 2. The safety score was 100 at $50 \%$ SOC since 4 out of 4 cells showed positive responses with no thermal runaway. The safety scored dropped to 50 at $60 \%$ SOC since 3 out of 6 cells showed negative responses and went to thermal runaway. At higher SOC, all the cells went to thermal runaway and with safety score of 0. Although the number of cells at each SOC could be more and the SOC levels could be in finer increments, this method demonstrated the possibility of comparing cells for safety and risk of thermal runaway. For example, the 100\% SOC 18Ahr LFP cells had the same safety risk of 50\% SOC 25Ahr NMC cells. At $60 \%$ SOC the NMC cells had a 50\% chance of going to thermal runaway compared to $0 \%$ for the LFP cells. Instead of a broad assessment of safety of the cells, this method provided a fair comparison among various cells under the same test conditions for mechanical abuse.

Table 2. NMC cells tested at various SOC.

\begin{tabular}{|c|c|c|c|c|c|c|c|}
\hline Cell & Voltage & Capacity & SOC & Number & Number of & Safety & Thermal \\
& $(\mathrm{V})$ & $($ Ahr $)$ & $(\%)$ & of Tests & thermal & Score & runaway \\
& & & & & runaway & & risk \\
\hline NMC & 3.68 & 12.5 & 50 & 4 & 0 & 100 & 0 \\
\cline { 2 - 8 } Cells & 3.71 & 15.0 & 60 & 6 & 3 & 50 & 50 \\
\hline
\end{tabular}




\begin{tabular}{|l|c|c|c|c|c|c|c|}
\hline & 4.00 & 20 & 80 & 1 & 1 & 0 & 100 \\
\cline { 2 - 8 } & 4.05 & 23 & 92 & 1 & 1 & 0 & 100 \\
\hline
\end{tabular}

Although there are some methods for battery safety assessment, such as those suggested by USABC [4], they are more appropriate for battery systems rather than individual cells. A simple "pass or fail" criteria used by previous mechanical abuse tests for consumer electronic cells is not an appropriate assessment for large-format cells and is probably the reason for limited adoption by the automotive industry. The analysis of the two types of commercial Li-ion cells showed it is possible to design mechanical abuse tests to compare safety risks of different large-format cells, using voltage drop characteristics and thermal runaway as parameters. However, further development of this method may require more detailed temperature response analysis.

For all the cells tested, temperature responses could be put in three categories:

1) Small temperature rise to $30-60{ }^{\circ} \mathrm{C}$ after short circuit

2) Large temperature rise from $60-130^{\circ} \mathrm{C}$ but no full scale thermal runaway

3) Temperature rise above $130{ }^{\circ} \mathrm{C}$ and followed by thermal runaway, fire and smoke The temperatures are an average over the entire cell surface. It should be noted in case 1) and 2) localized temperature rise could exceed the range. The overall temperatures of the cell remain in the defined range. It is also important to combine the temperatures with the voltage response. More detailed distinctions based on cell responses are possible, but may not be practical, especially in cell production environments. Therefore, a refined and simplified thermal runaway risk (TRR) assessment is proposed without the use of post-mortem 
examinations. The TRR score is only based on two continuously measured parameters during and after the mechanical abuse test: 1) cell voltage and 2) surface temperature. In the TRR assessment score system:

- TRR 0 or safety score of 100: "Soft short", voltage drop-and-recovery and average temperature rise $<60^{\circ} \mathrm{C}$, which has no loss of cell function and posts no thermal hazard, comparable to USABC Level 0 or Level 1

- TRR 50 Safety score of 50: Continuous voltage decrease, without recovery, average temperature rise between $60-130^{\circ} \mathrm{C}$, which represents permanent cell damage and thermal hazard comparable to USABC Levels 2-3.

- TRR 100 and safety score of 0: Quick drop of voltage and fast rise of temperature > $130^{\circ} \mathrm{C}$ within $3-5$ seconds. It includes various features of thermal runaway including venting and mass loss, pouch rupture, and sometimes fire and explosion, comparable to USABC Level 4 to Level 7.

It should be pointed out that for large format Li-ion cells, the second condition may not be common because of the difficulty in controlling the actually failure size and the amount of energy release. A well designed test system should have significant number of cells in between TRR scores of 100 and 0 . In the overall safety assessment, four or more cells should be tested in each condition, and each cell carries the same weight in the final score. The thermal runaway risks listed in Table 1 and 2 represent the sum of weighted TRR under each SOC. It is therefore possible to use this score system to develop mechanical abuse tests to evaluate large-format Li-ion cells. 


\section{Summary}

Internal short circuit simulation was expanded from testing small prismatic cells to mechanical abuse tests of large format cells used in electric vehicles. The original pinch test introduced larger damages and prevented testing of higher capacity cells or cells at high stateof-charge. Significant efforts have been put to modify the pinch test. It was found adding a torsion component to the pinch test could produce separator failure at smaller load thus minimize the short circuit size. A modified pinch-torsion test was used to test fully charged large-format cells. The study was able to conclude that 18 Ahr LFP cells have higher TRR scores than the 25 Ahr NMC cells. Since the purpose of this study was to compare two commercial cells with similar physical dimensions but different cell chemistries, the pinchtorsion test was sensitive enough to distinguish these two types of cells. This test method can be further optimized to evaluate Li-ion cells used in electric vehicle applications.

\section{ACKNOWLEDGEMENT}

The authors would like to thank Dr. James Barnes for his support of the project and Dr.

Donald Erdman for building the test rig. This work was sponsored by the Office of Vehicle Technologies of the Department of Energy and was carried out at Oak Ridge National Laboratory under contract DE-AC05-00OR22725 with UT-Battelle, LLC.

\section{References}

[1] A.J. Ilott, M. Mohammadi, H.J. Chang, C.P. Greyand, A. Jerschow, PNAS, Vol. 113 No. 39, (2016) 10779-10784

[2] Mahmood Tabaddor, "New Internal Short Circuit Test Development for Battery Safety 
Standards", UL presentation at 2011 U.S.-China Electric Vehicle and Battery Technology

Workshop, http://www.cse.anl.gov/us-china-

workshop/2011/pdfs/batteries/2011\%20US\%20China\%20EV\%20Workshop\%20on\%20ISC \%20at\%20UL.PDF, 2011

[3] Mehmet Rona, YooEup Hyung, Brian Barnett and Suresh Sriramulu, "The Relationship of the Nail Penetration Test to Safety of Li-Ion Cells", DOE 2013 Merit Review, http://www4.eere.energy.gov/vehiclesandfuels/resources/merit/review/sites/default/files/es14 2_sriramulu_2013_p.pdf, May 2013.

[4] UL1642, “UL Standard for Safety for Lithium Batteries”, ISBN 1-55989-829-1, 1999

[5] C. N. Ashtiani, ECS Transactions, 11 (19) (2008) pp1-11

[6] Battery Association of Japan, Presentation at UN Informal Working Group Meeting, November 11-13, 2008, Washington, DC.

[7] C. J. Orendorff, E. P. Roth, G. Nagasubramanian, Journal of Power Source, 196(15) (2011) pp6554-6558

[8] M. Keyser, D. Long, Y. S. Jung, A. Pesaran, E. Darcy, B. McCarthy, L. Patrick, and C. Kruger, "Development of a Novel Test Method for On-Demand Internal Short Circuit in a LiIon Cell”, Large Lithium Ion Battery Technology and Application Symposium Advanced Automotive Battery Conference, http://www.nrel.gov/vehiclesandfuels/energystorage/pdfs/50082.pdf Jan 25-28, 2011, Pasadena, CA

[9] C. Orendorff, et al., ECS Meeting, May 2009

[10] C. Orendorffand E.P. Roth, USABC TT Meeting, Feb 2009 
[11] C. Orendorff, et al., ECS Meeting, Oct 2010

[12] H. Maleki, J.N. Howard, J. Power Sources 191 (2009) 568-574.

[13] W. Cai, H. Wang, H. Maleki, et al. J. Power Sources 196 (2011) 7779-7783.

[

[14] F. Ren, T. Cox and H. Wang, Journal of Power Sources Vol.249, (2014) pp156-162

[15] Yuzhu Xia, Tianglei Li, Fei Ren, Yanfei Gao and H. Wang, Journal of Power Sources, Vol 256C, (2014) pp356-362 